\title{
Effects of exercise on the expression of long non-coding RNAs in the bone of mice with osteoporosis
}

\author{
JIANMIN GUO ${ }^{1}$, YU YUAN ${ }^{1,2}$, LINGLI ZHANG ${ }^{1,2}$, MIAO WANG $^{1}$, XIAOYANG TONG ${ }^{1}$, \\ LIFEI LIU ${ }^{1}$, MIAO ZHANG ${ }^{1}$, HUI LI ${ }^{1}, \mathrm{XI} \mathrm{CHEN}^{1,3}$ and JUN ZOU ${ }^{1}$ \\ ${ }^{1}$ School of Kinesiology, Shanghai University of Sport, Shanghai 200438; ${ }^{2}$ School of Physical Education \\ and Sports Science, South China Normal University, Guangzhou, Guangdong 510631; \\ ${ }^{3}$ School of Sports Science, Wenzhou Medical University, Wenzhou, Zhejiang 325003, P.R. China
}

Received June 23, 2021; Accepted September 22, 2021

DOI: $10.3892 / \mathrm{etm} .2021 .10993$

\begin{abstract}
Physical activity or exercise are known to promote bone formation and decrease bone resorption to maintain skeletal and bone health both in animal models and in humans with osteoporosis. Previous studies have indicated that long non-coding RNAs (IncRNAs) are able to regulate bone metabolism. Therefore, the present study aimed to evaluate whether lncRNAs responded to exercise by regulating the balance of bone metabolism in order to prevent osteoporosis. To meet this end, ovariectomized mice were used in the present study to establish an osteoporosis model. The exercise treatment groups were subjected to 9 weeks of treadmill running exercise in 4 weeks of the operation was performed Femurs were collected to measure bone mineral density, bone mass, bone formation and resorption. The expression levels of lncRNAs were subsequently measured using microarray and gene function analyses. The pairwise comparison results [ovariectomy (OVX) vs. OVX + exercise (EX); OVX vs. SHAM; SHAM vs. $\mathrm{SHAM}+\mathrm{EX} ; \mathrm{OVX}+\mathrm{EX}$ vs. SHAM + EX] of the gene microarray analysis revealed that the expression of 2,424 lncRNAs (1718 upregulated and 706 downregulated) were significantly altered in the mouse femurs following treadmill running. Gene Ontology (GO) analysis, incorporating the GO annotations 'biological processes', 'molecular function' and 'cellular components', of osteoporosis revealed that the VEGF, mTOR and NF- $\mathrm{NB}$ signaling pathways were potential targets of the lncRNAs. Moreover, it was possible to predict the target microRNAs (miRNAs) of six lncRNAs (LOC105246953,
\end{abstract}

Correspondence to: Professor Jun Zou, School of Kinesiology, Shanghai University of Sport, 339 Changhai Road, Shanghai 200438, P.R. China

E-mail: junzou@sus.edu.cn

Dr Xi Chen, School of Sports Science, Wenzhou Medical University, 270 West Xueyuan Road, Wenzhou, Zhejiang 325003, P.R. China

E-mail: chenab004@126.com

Key words: exercise, osteoporosis, long non-coding RNA, microRNA
LOC102637959, NONMMUT014677, NONMMUT027251, rilD130079K21IPX00187K1611491 and NONMMUT006626), which suggested that the underlying mechanism by which lncRNAs respond to exercise involved bone regulation via IncRNA-miRNA sponge adsorption. Overall, these results suggested that the treadmill running exercise did regulate lncRNA expression in the bone, and that this was involved in the prevention of osteoporosis.

\section{Introduction}

Osteoporosis is an age-associated metabolic bone disease that is characterized by decreasing bone mass and deteriorating bone microstructure, leading to a general loss in bone mineral density (BMD) (1). Appropriate exercise is generally recommended for the prophylaxis and treatment of osteoporosis, especially high-impact exercise (2,3). Exercise increases bone formation and reduces bone resorption, thereby improving bone mass and strength in aging individuals (4). Furthermore, exercise has been demonstrated to increase peak bone mass in growing mice (5). However, the underlying mechanism has yet to be fully elucidated.

Previous studies have demonstrated that mechanical loading and hormones are the factors that are predominantly associated with the effects of exercise on the regulation of bone homeostasis; and non-coding RNAs, including microRNAs (miRNAs/miRs), have been reported to be involved in this process (6-8). Long non-coding RNAs (lncRNAs) are another type of non-coding RNA, and these are characterized by having lengths of $>200 \mathrm{bp}$ (9). LncRNAs differ from miRNAs by having more complex structures and functions due to their longer sequences (10). An increasing number of studies have revealed that IncRNAs participate in several physiological processes of bone cells; in particular, exerting roles in cell proliferation and differentiation by regulating gene expression (11-13). The lncRNA differentiation antagonizing non-protein coding RNA has been demonstrated to inhibit the differentiation of bone marrow-derived mesenchymal stem cells (BMSCs) via inactivation of the p38/mitogen-activated protein kinase (MAPK) signaling pathway (14). Knockdown of the lncRNA MIR31HG leads to an increase in the osteogenesis of adipose-derived stem cells (15). Furthermore, 
lncRNAs may regulate gene transcription by interacting with miRNAs in osteoclasts and osteoblasts, thereby influencing their activity (16). For example, the lncRNA potassium voltage-gated channel subfamily KCNQ1 has been indicated to inhibit osteolysis by inhibiting miR-21a-5p in bone marrow-derived macrophages (17), and the lncRNA TSIX transcript, XIST antisense RNA, promotes osteoblast apoptosis by inhibiting miR-30a-5p (18). These studies have demonstrated that lncRNAs are involved in bone modeling, and may be key factors affecting osteoporosis development.

Therefore, the present study aimed to investigate whether exercise is able to regulate lncRNA expression, thereby affecting bone remodeling. In addition, evidence was sought after for an improved understanding of the underlying mechanism by which osteoporosis is prevented through exercise.

\section{Materials and methods}

Animals and establishment of the animal model. The present study was approved by the Ethics Committee of Shanghai University of Sport (approval no. 2015030; Shanghai, China), and all procedures were conducted following the recommendations of the ARRIVE guidelines (19). The ovariectomized (OVX) mouse was used to establish osteoporosis model due to estrogen deficiency (20). A total of 44 C57BL/6 female mice (age, 12 weeks old; weight, 22-24 g; provided by Changzhou Cavens Laboratory Animal Co., Ltd; http://www.cavens.com.cn/) were used in the study and randomly divided into four groups $(\mathrm{n}=11$ mice in each group) as follows: i) The ovariectomy (OVX) group; ii) the sham operation (SHAM) group; iii) the ovariectomy and exercise (OVX + EX) group; and iv) the sham operation and exercise $(\mathrm{SHAM}+\mathrm{EX})$ group. The mice in the sham group received a sham operation consisting in the removal of an equal size of fat around the uterus. The mice in the OVX group received a bilateral ovariectomy. Before the operations, mice were anesthetized with avertin $(300 \mathrm{mg} / \mathrm{kg})$. All experimental mice were housed in an environment with a 12-h light/dark cycle at a temperature of $22 \pm 3^{\circ} \mathrm{C}$ and a humidity of $55-60 \%$, and they were provided with food and water ad libitum. The mice were weighed on Monday each week.

Exercise protocol. The two exercise groups of mice (the OVX $+\mathrm{EX}$ and SHAM + EX groups) were subjected to 9 weeks of treadmill running exercise 4 weeks after the operation was performed ( $\mathrm{n}=11$ mice in each group). The treadmill running training program was adhered to as described in previous studies $(7,21)$, with the following modifications. In brief, the speed was set at $6 \mathrm{~m} / \mathrm{min}$ for $30 \mathrm{~min}$ for the 1 st week (which comprised 5 days of exercise followed by 2 days of rest) to allow the mice to become familiarized with the exercise regime. From the 2 nd week, all the mice performed a $5 \mathrm{~min}$ warm-up exercise at a speed of $6 \mathrm{~m} / \mathrm{min}$ with a $25^{\circ}$ slope, followed by a 55 min running exercise at a speed of $8 \mathrm{~m} / \mathrm{min}$ with a $25^{\circ}$ slope. Subsequently, the running exercise speed was increased by $1 \mathrm{~m} / \mathrm{min}$ each week leading up to the completion of the treadmill running program after a total of 9 weeks, culminating in a running speed of $15 \mathrm{~m} / \mathrm{min}$ with a $25^{\circ}$ slope for the last training week (Fig. 1A). The OVX and SHAM groups were housed under conventional conditions without the treadmill running training program as a control.
Tissue preparation. All mice were subcutaneously injected with calcein $(0.01 \mathrm{mg} / \mathrm{g}$ weight $)$ to fluorescently label the bones at 8 and 2 days prior to sacrifice. The mice were sacrificed to collect bone samples $48 \mathrm{~h}$ after the last treadmill training session. Briefly, all mice were anesthetized with avertin $(300 \mathrm{mg} / \mathrm{kg})$, and the animals were sacrificed by $\mathrm{CO}_{2}$ asphyxiation (flow rate of $\mathrm{CO}_{2}, 2 \mathrm{l} / \mathrm{min}$; air displacement rate, 20\%/min). Death was confirmed by the absence of breathing, response to a firm toe pinch, absence of a heartbeat or respiratory sounds, graying of the mucous membranes and rigor mortis. The animal ethics approval was obtained in November 2015, and these experiments were performed in March 2016. Femurs were collected from all the mice to subsequently perform microarray analysis of the lncRNAs, microcomputed tomography ( $\mu \mathrm{CT})$, dual-energy X-ray absorptiometry (to determine the BMD) and bone biomechanics and bone histomorphometry analyses.

Microarray analysis. A total of three right femurs were selected from each of the four groups for total RNA extraction using Invitrogen TRIzol ${ }^{\circledR}$ reagent (Thermo Fisher Scientific, Inc.), and the extracted RNA was reverse-transcribed into cDNA according to the manufacturer's protocol (RevertAid First Strand cDNA Synthesis kit; cat. no. K1622; Thermo Fisher Scientific, Inc.). Subsequently, a lncRNA Mouse Gene Expression Microarray system V1.0 (microarray and service provided by Boao Biological Group Co., Ltd.) was used to detect of the expression levels of lncRNAs. Sample labeling and array hybridization were performed strictly according to the manufacturer's protocol (One-Color RNA Spike-In kit; cat. no. 5188-5282; Agilent,). G2565CA Microarray Scanner (Agilent Technologies, Inc.) was used to scan the chip. Feature Extraction software v10.7 (Agilent Technologies, Inc.) was used to extract the data from the resulting images, and these data were subsequently analyzed using GeneSpring software v11.5 (Agilent Technologies, Inc.). The filter criteria were set as fold-change $\geq 2$ and $\mathrm{P}<0.05$ for determination of the differential expression of IncRNAs between groups. The datasets generated and/or analyzed during the current study are available in the Gene Expression Omnibus repository, https://www. ncbi.nlm.nih.gov/geo/query/acc.cgi?acc=GSE184226.

Gene function analysis. Gene Ontology (GO) (http://www. geneontology.org) and Kyoto Encyclopedia of Genes and Genomes (KEGG; https://www.kegg.jp/) analyses (consulted in May, 2016) were performed to investigate the underlying 'molecular function', 'biological process', 'cellular component' and pathway terms of the genes of interest. The significance of the GO term enrichment and KEGG pathway correlations were assessed according to the enrichment score yield based on the P-value.

Six of the 18 co-expressed lncRNAs in the OVX + EX group and SHAM group (compared with the OVX group) were selected for target microRNAs analysis. Target miRNA prediction analysis was performed using the program RegRNA 2.0 (http://regrna2.mbc.nctu.edu.tw/detection.html) based on gene sequence.

$\mu C T$ analysis. A total of three left femurs from each of the four groups were scanned using a $\mu \mathrm{CT}$ device (SkyScan $1174 \mathrm{v} 2$; Bruker-microCT; Bruker Corporation) using the following 


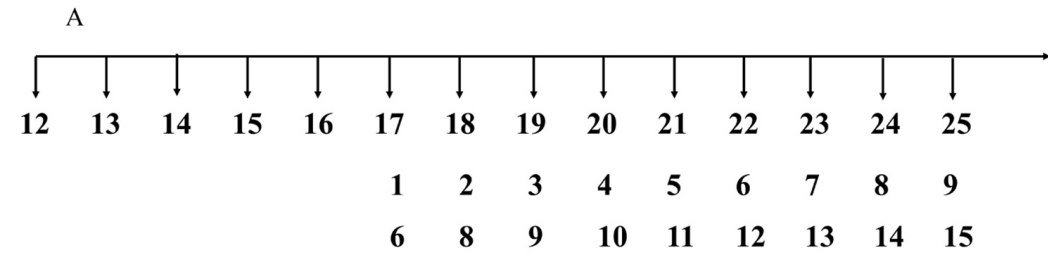

30

B

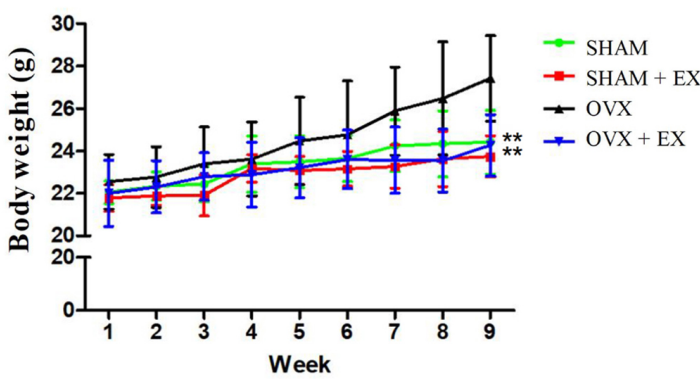

$\mathrm{D}$
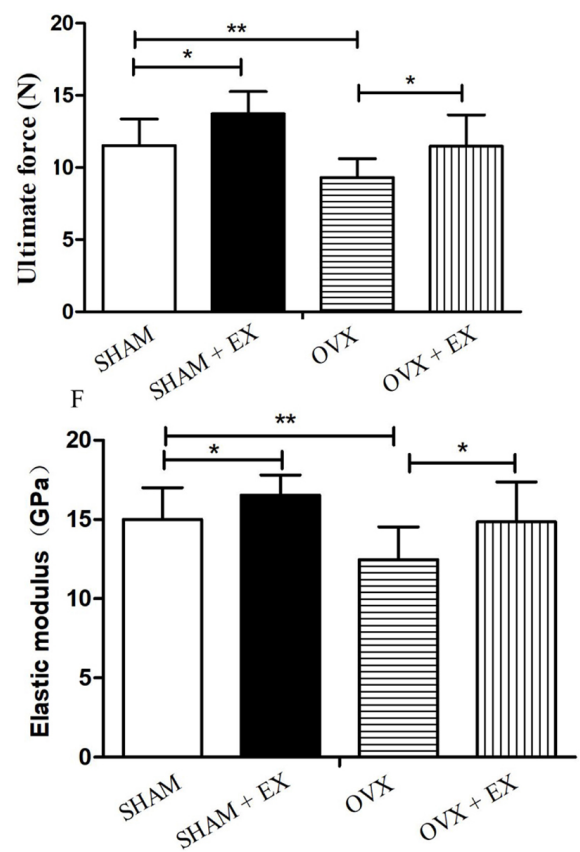

55

$\mathrm{C}$

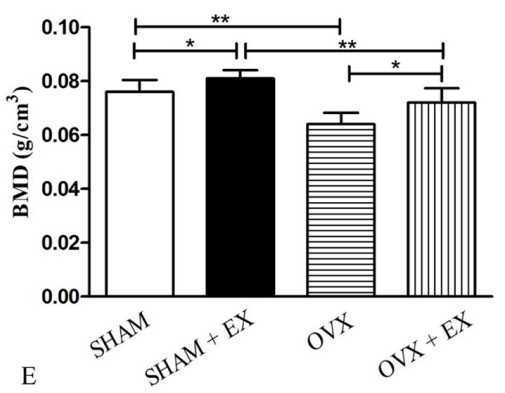

$\square$ SHAM

- SHAM + EX

曰 ovx

皿 $\mathrm{OVX}+\mathrm{EX}$

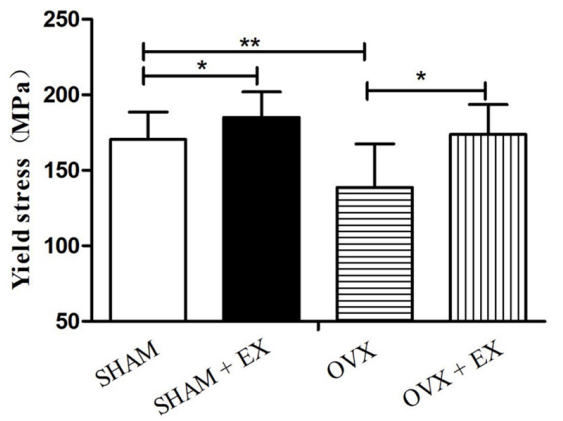

Figure 1. Effects of exercise and/or ovariectomy on body weight, BMD and bone biomechanics. (A) Outline of the exercise protocol. (B) Body weight of mice in each group ( $\mathrm{n}=11$ mice). ${ }^{* *} \mathrm{P}<0.01$, the SHAM group or the OVX + EX group vs. the OVX group. (C) BMD of mice in each group (n=8). (D) Effect of exercise on bone ultimate force ( $\mathrm{n}=8$ mice). (E) Effect of exercise on bone yield stress $(\mathrm{n}=8)$. (F) Effect of exercise on bone elastic modulus $(\mathrm{n}=8)$. ${ }^{*} \mathrm{P}<0.05$; ${ }^{* *} \mathrm{P}<0.01$. OVX, bilateral ovariectomy group; OVX + EX, bilateral ovariectomy and exercise group; SHAM, sham operation group; SHAM + EX, sham operation and exercise group. BMD, bone mineral density.

settings: $9.26 \mu \mathrm{m}$ Camera pixel size; $46 \mathrm{kV}$ voltage; and a current of $800 \mu \mathrm{A}$. A total of $125 \mu \mathrm{CT}$ slices were acquired as regions of interest for further analysis. The trabecular number (Tb.N; mm), bone volume/tissue volume (BV/TV, \%), trabecular separation (Tb.Sp; $\mathrm{mm}$ ) and trabecular thickness (Tb.Th; $\mathrm{mm}$ ) were then calculated using the software provided by the $\mu \mathrm{CT}$ system.

BMD and bone biomechanical analyses. Dual-energy X-ray absorptiometry, using an Osteocore 3 Digital 2D bone densitometer (Medilink), was used to detect the BMD of the left femurs (eight samples analyzed for each group), followed by a bone biomechanical test. Femurs were thawed at $4{ }^{\circ} \mathrm{C}$ in normal saline solution and carefully checked under a light microscope (Leica Microsystems GmbH; magnification, x10) to confirm the integrity of all bones before the test was performed.

The three-point bending method was used to detect the mechanical indices of all bones (22). Both proximal and distal areas of the left femur were fixed on two supports of the machine with a $10-\mathrm{mm}$ span. The front side of the femur was placed facing upwards. A probe was then moved down at a speed of $1 \mathrm{~mm} / \mathrm{min}$ with no pre-load, and the movement of the probe was allowed to proceed downwards for $1 \mathrm{~min}$ after the bone fracture. The software (LabSANS-Test D30C) enabled 

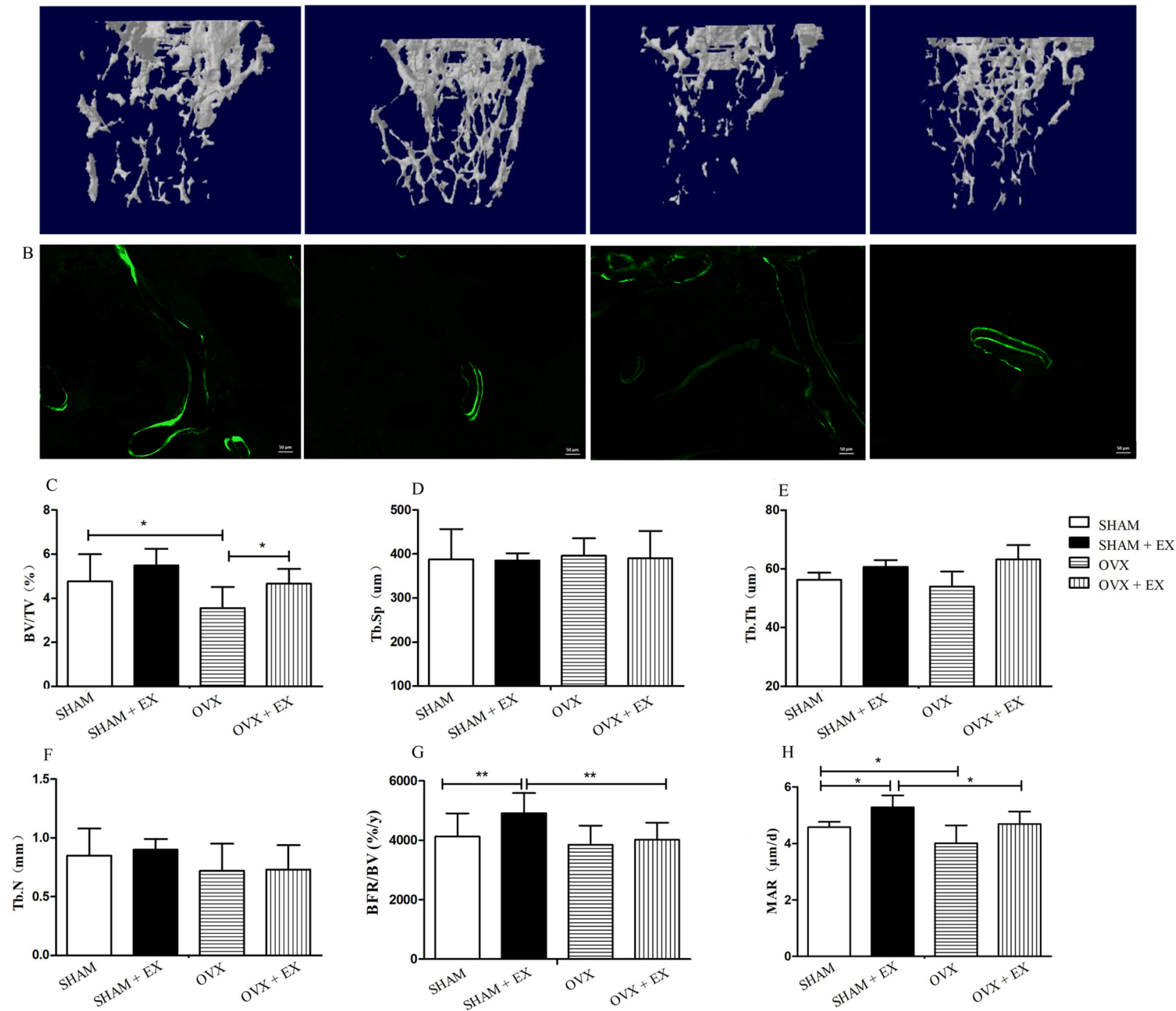

Figure 2. Results of $\mu \mathrm{CT}$ and effect of exercise on bone histomorphometric parameters. (A) Representative $\mu \mathrm{CT}$ images demonstrating the three-dimensional trabecular architecture in mouse femur. (B) Representative images of mouse bones which were labeled with subcutaneously injected calcein (5 $\mu 1 / \mathrm{g})$ at days 1 and 8. (C) Trabecular bone mass (BV/TV), (D) Tb.Sp, (E) Tb.Th and (F) Tb.N of mouse femur was determined using $\mu$ CT. (G) Effect of exercise on BFR/BV. (H) Effect of exercise on the MAR. ${ }^{*} \mathrm{P}<0.05 ;{ }^{* *} \mathrm{P}<0.01$. OVX, bilateral ovariectomy group; OVX + EX, bilateral ovariectomy and exercise group; SHAM, sham operation group; SHAM + EX, sham operation and exercise group; $\mu \mathrm{CT}$, microcomputed tomography; MAR, mineral apposition rate; BV/TV, bone volume/ tissue volume; BFR/BV, bone formation rate/bone volume; Tb.N, trabecular number; Tb.Sp, trabecular separation. Tb.Th, trabecular thickness.

calculation of the mechanical indices of the bones, including the elastic modulus $(\mathrm{GPa})$, yield stress $(\mathrm{MPa})$ and ultimate force $(\mathrm{N})$.

Bone histomorphometry. The right femurs were fixed with $4 \%$ paraformaldehyde at room temperature for $24 \mathrm{~h}$, and resin embedding was used for the bone histomorphometry test. Following the procedure outlined in a previous study (23), the right distal femurs were dehydrated in an increasing series of ethanol (percentages of 70, 95 and 100\%), cleared using xylene and embedded in glycol methacrylate. The samples were then cut into $4-\mu$ m-thick slices using a ultra-microtome (Leica Microsystems GmbH). After which, one of the maximum-profile slices of each sample was selected for further analysis. The slice was directly examined using double labeling with calcein by using fluorescence microscopy (Leica Microsystems GmbH; magnification, x10) to detect the mineral apposition rate (MAR; percentage of cancellous bone MAR) and, the bone formation rate/bone volume (BFR/BV) using a digitizing morphometric system (Osteomeasure High Resolution Color Subsystem; Osteometrics, Inc.).

Statistical analysis. All data are presented as the mean \pm SD. A one-way ANOVA was performed, followed with a Bonferroni's post hoc test to analyze the effects of exercise or bilateral ovariectomy on the bones of mice. All statistical analyses were performed using SPSS statistical software v20.0 (IBM Corp.), and the figures were prepared using GraphPad Prism software v5.0 (GraphPad Software, Inc.). $\mathrm{P}<0.05$ was considered to indicate a statistically significant difference. 

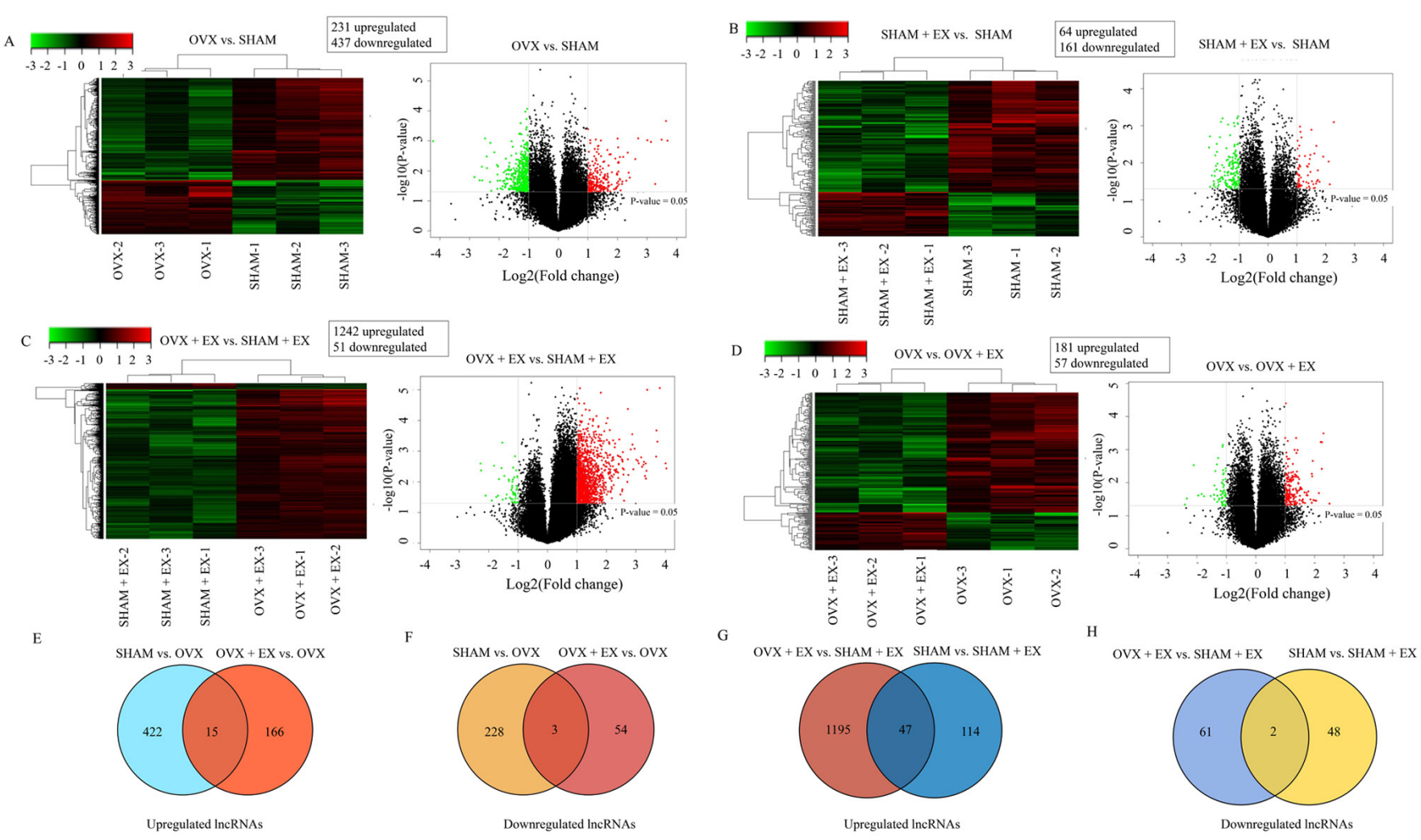

Figure 3. Effect of exercise on the expression profiles of differentially expressed lncRNAs in the bone of mice. Hierarchical cluster of lncRNA expression profiles: (A) OVX vs. SHAM, (B) SHAM + EX vs. SHAM, (C) OVX + EX vs. SHAM + EX and (D) OVX vs. OVX + EX. (E and F) Co-expression of lncRNAs in the OVX + EX and SHAM groups that are (E) upregulated or (F) downregulated compared with the OVX group. (G and $\mathrm{H})$ Co-expression of lncRNAs in the OVX + EX and SHAM groups that are $(\mathrm{G})$ upregulated or $(\mathrm{H})$ downregulated compared with the SHAM group. OVX, bilateral ovariectomy group; OVX + EX, bilateral ovariectomy and exercise group; SHAM, sham operation group; SHAM + EX, sham operation and exercise group; lncRNA, long non-coding RNA.

\section{Results}

Effect of exercise on the animals' weight, BMD and bone strength. No significant differences in body weight were observed among the four groups at the beginning of the experiment. However, after 9 weeks of training, the body weight of the OVX group was significantly increased compared with the SHAM group $(\mathrm{P}<0.01)$, while treadmill running significantly decreased the body weight of the OVX + EX group $(\mathrm{P}<0.01)$. However, no significant differences in weight were observed between the SHAM and SHAM + EX groups (Fig. 1B).

The OVX group was revealed to have a significantly lower BMD compared with the SHAM group $(\mathrm{P}<0.01$; Fig. $1 \mathrm{C})$. The exercise training regime was demonstrated to increase the BMD, as the SHAM + EX and OVX + EX groups had significantly higher BMDs compared with the SHAM and OVX groups, respectively (both $\mathrm{P}<0.05$; Fig. 1C). In addition, the ultimate force, elastic modulus and yield stress measurements of the OVX group were all significantly lower compared with those of the SHAM group (all P $<0.01$; Fig. 1D-F). The OVX + EX group also had significantly increased bone strength compared with the OVX group, as determined from the ultimate force, elastic modulus and yield stress values (all $\mathrm{P}<0.05$ ); while the SHAM + EX group also had significantly increased ultimate force, elastic modulus and yield stress values compared with the SHAM group $(\mathrm{P}<0.05$; Fig. 1D-F). In summary, the OVX group mice were identified as having lower BMDs and bone strength values than the mice in the
SHAM group, which confirmed that the osteoporosis animal model had been successfully established in the present study. Exercise increased the BMD and the bone strength of the OVX group, which indicated that exercise played a role in the prevention and treatment of osteoporosis.

Effect of exercise on bone mass, bone formation. The results of the $\mu \mathrm{CT}$ analysis revealed no significant differences in the values of Tb.N, BV/TV, Tb.Sp and Tb.Th between the SHAM and SHAM + EX groups. In addition, the BV/TV ratio was increased in the OVX + EX group compared with the OVX group $(\mathrm{P}<0.05$; Fig. 2C-F).

The results of the bone histomorphometry analysis revealed that the OVX group had a lower MAR compared with the SHAM group $(\mathrm{P}<0.05)$. However, 9 weeks of exercise training in the SHAM + EX group led to a significant increase in BFR/BV and MAR values compared with the SHAM group (both $\mathrm{P}<0.05$; Fig. $2 \mathrm{G}$ and $\mathrm{H}$ ). Though exercise increased the $\mathrm{BFR} / \mathrm{BV}$ and MAR values in the OVX + EX group, there was no significant difference. In summary, the above results indicated that exercise promoted the bone formation and bone mass of OVX mice, thereby preventing and treating osteoporosis.

Effect of exercise on the expression of IncRNAs. The results of the present study indicated that the establishment of the ovariectomy model altered the patterns of IncRNA expression in mice. A total of $231 \mathrm{lncRNAs}$ were significantly upregulated and 437 were significantly downregulated in the bones 

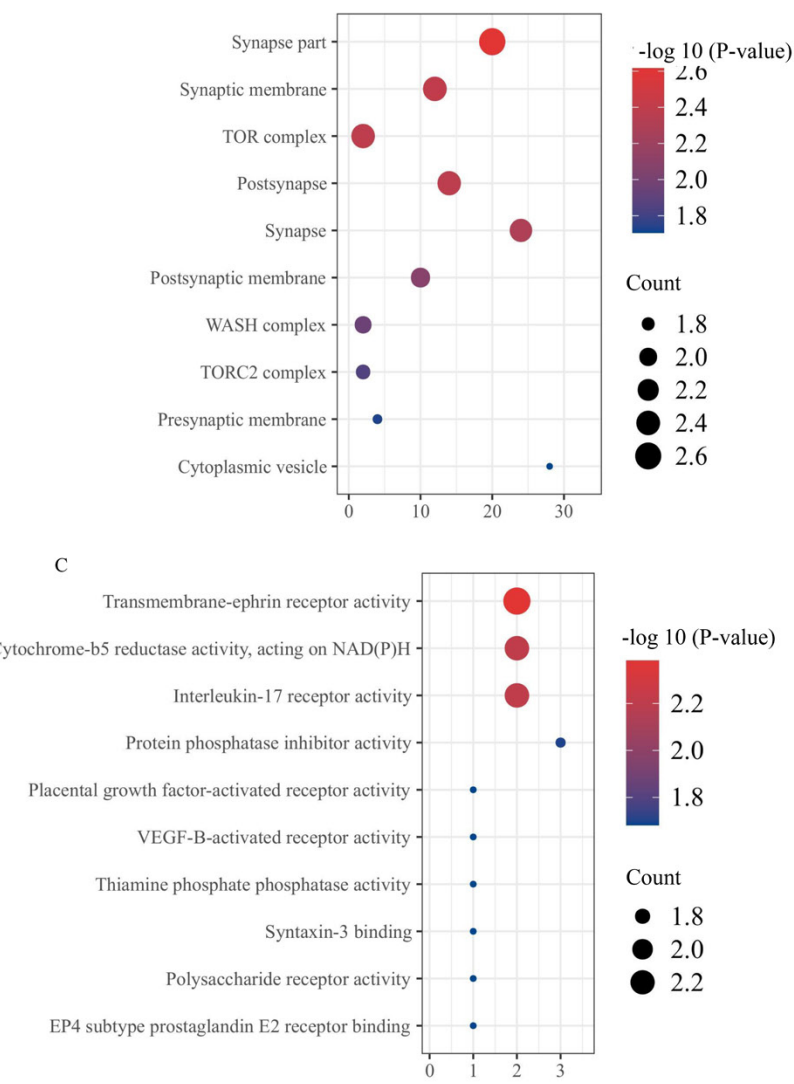

B
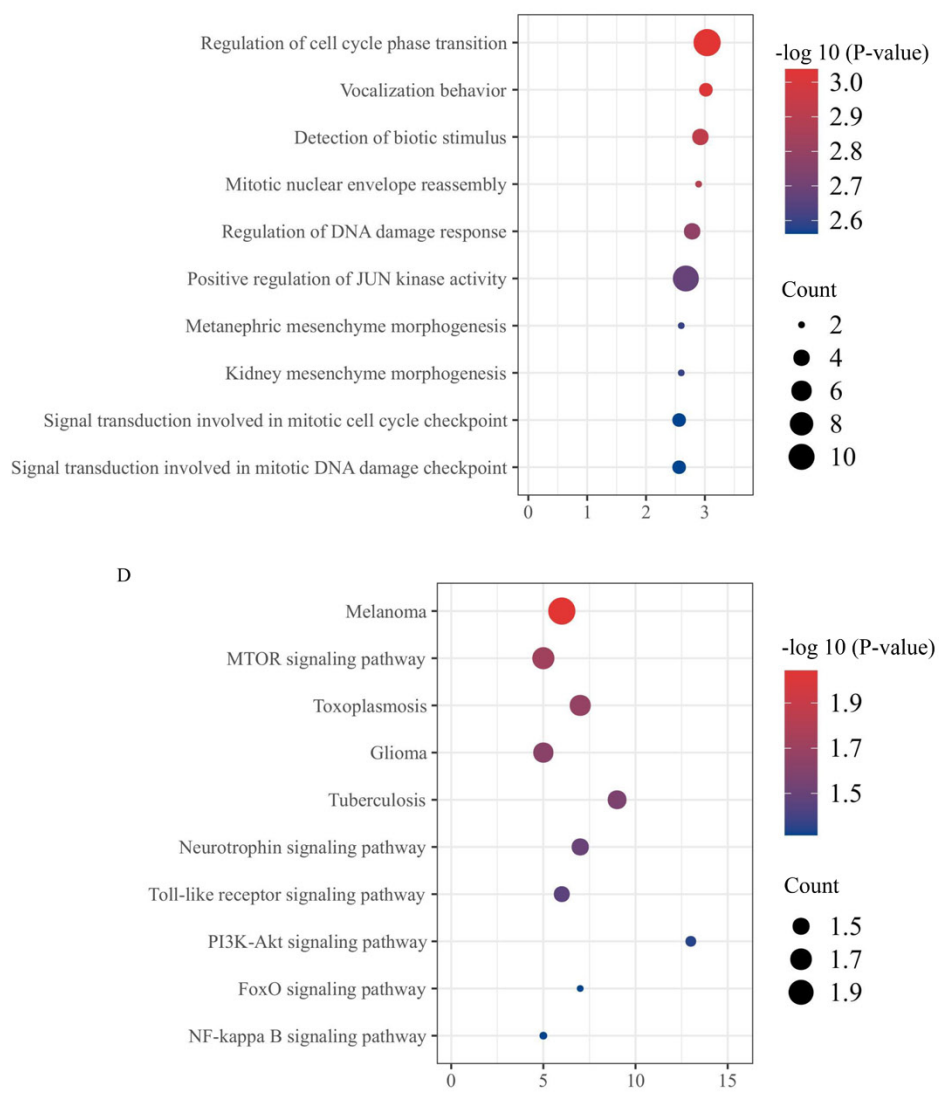

Figure 4. GO and KEGG pathway analysis of the biological functions of genes co-expression with differentially expressed lncRNAs. Top 10 (A) 'cellular component', (B) 'biological process' and (C) 'molecular function' terms of the differentially expressed lncRNAs in the OVX group compared with the OVX + EX group. (D) Top 10 pathways of the differentially expressed lncRNAs in the OVX group compared with the OVX + EX group. GO, Gene Ontology; KEGG, Kyoto Encyclopedia of Genes and Genomes; lncRNA, long non-coding RNA.

of the OVX group compared with the SHAM group (Fig. 3A). Furthermore, the treadmill running exercise regime also led to further changes in the expression of lncRNAs in the bone. A total of 64 lncRNAs were significantly upregulated and $161 \mathrm{lncRNAs}$ were significantly downregulated in the bones of the SHAM + EX group compared with the SHAM group (Fig. 3B). A total of 1,242 lncRNAs were significantly upregulated and 51 lncRNAs were significantly downregulated in the bones of the OVX + EX group compared with the SHAM + EX group. (Fig. 3C). Additionally, it was revealed that 181 lncRNAs were significantly upregulated and 57 lncRNAs were downregulated in the bones of the OVX + EX group compared with the OVX group (Fig. 3D), and lncRNA H19 was among the upregulated lncRNAs (fold change, 2.1; $\mathrm{P}=0.027$ ). Finally, it was observed that 15 upregulated and three downregulated IncRNAs were common amongst the OVX + EX and SHAM groups when compared against the OVX group (Fig. 3E and F). Moreover, it was also observed that 47 upregulated and two downregulated lncRNAs were common amongst the OVX + EX and SHAM groups when compared with the SHAM + EX group (Fig. 3G-H). The above results demonstrated that exercise altered the expression of some lncRNAs in the bone of osteoporotic mice, which indicated that exercise may play a role in preventing and treating osteoporosis by regulating lncRNAs.
GO enrichment and KEGG analysis. GO analyses of the lncRNAs in the OVX +EX and OVX groups were performed to compare them, and also to investigate how IncRNAs regulated gene transcription. The bioinformatics analysis in the present study revealed that the IncRNAs in these groups were associated with the 'cellular components', 'biological processes' and 'molecular functions' annotations, the top ten results obtained from the GO enrichment analysis of the differential genes are presented in Fig. 4A-C. 'Synapse part' and 'TOR complex' were among the top 10 enriched terms of cellular components; 'regulation of cell cycle phase transition' and 'positive regulation of JUN kinase activity' were among the top 10 enriched terms of biological processes; and 'VEGF-B-activated receptor activity' and 'interleukin-17 receptor activity' were among the top ten enriched terms of molecular functions (Fig. 4A-C). Subsequently, the KEGG analysis revealed that the 'mTOR', 'NF-kB' and 'PI3K/Akt' signaling pathways were likely to be involved in the mechanism through which the treadmill running exercise regime was able to exert its influence on the prevention and treatment of the osteoporosis process (Fig. 4D). The above results indicated that lncRNAs may promote bone formation through the above-mentioned factors or signal pathways, such as VEGF-B and the PI3K/Akt signaling pathway.

Target miRNAs of the IncRNAs. IncRNAs that were downregulated in the OVX group compared with the SHAM group, 
Table I. Fold changes of the co-expressed lncRNAs in the $\mathrm{OVX}+\mathrm{EX}$ and SHAM groups that are upregulated or downregulated compared with the OVX group.

A, Upregulated lncRNAs

\begin{tabular}{lll}
\hline Gene symbol & $\begin{array}{c}\text { FC of } \\
\text { OVX + EX }\end{array}$ & $\begin{array}{l}\text { FC of } \\
\text { SHAM }\end{array}$ \\
\hline Gm35194 & 2.15 & 2.37 \\
LOC105246953 & 2.44 & 2.33 \\
LOC102637959 & 2.08 & 2.07 \\
Gm30392 & 2.64 & 2.72 \\
NONMMUT014677 & 2.51 & 2.08 \\
NONMMUT016039 & 2.14 & 3.17 \\
NONMMUT027251 & 2.61 & 3 \\
NONMMUT067810 & 2.37 & 4.1 \\
ril7330424C03IPX00650H15|3890 & 2.04 & 2.14 \\
ril8430440M04|PX00025O03|1121 & 2.05 & 2.49 \\
ril9430019C24IPX00108O13|1178 & 2.11 & 2.42 \\
rilA230069F12IPX00129C15|2187 & 2.4 & 2.24 \\
rilA430104H18|PX00064B06|3523 & 2.05 & 2.39 \\
rilB020006F18IPX00325E01I1577 & 2.87 & 3.02 \\
rilF930011C10IPL00010K14|3597 & 2.23 & 3 \\
\hline
\end{tabular}

B, Downregulated lncRNAs

\begin{tabular}{lcc}
\hline Gene symbol & $\begin{array}{c}\text { FC of } \\
\text { OVX + EX }\end{array}$ & $\begin{array}{c}\text { FC of } \\
\text { SHAM }\end{array}$ \\
\hline NONMMUT006626 & 2.17 & 2.54 \\
rilD130079K21IPX00187K1611491 & 2 & 2.35 \\
uc.mouse.68 & 3.87 & 2.75 \\
\hline
\end{tabular}

OVX, ovariectomy; EX, exercise; lncRNA, long non-coding RNA; FC, Fold change vs. the OVX group.

and whose expression levels were also partially reversed in the OVX + EX group, were selected for further study. Among the 15 upregulated and three downregulated lncRNAs (Fig. 3E and F; Table I), four upregulated lncRNAs and two downregulated lncRNAs were randomly selected to perform miRNA prediction analysis using the program RegRNA 2.0. A total of 43 miRNAs were identified as being the potential targets of the six selected lncRNAs (Table II). The present results indicated that exercise may alter the expression of specific lncRNAs, thereby enhancing their inhibition of targeted miRNAs to promote bone formation.

\section{Discussion}

The OVX group mice were identified as having lower BMDs and bone strength values than the mice in the SHAM group, which confirmed that the osteoporosis animal model had been successfully established in the present study. Although 9 weeks of treadmill running exercise did reverse the bone loss and prevent osteoporosis in the ovariectomized mice, as was anticipated, the results revealed that the OVX + EX group had higher BMD and bone strength values compared with the OVX group, and these findings were consistent with those of a previous study (7). A previous study also demonstrated that 10 weeks of treadmill training reduces the bone-resorbing in ovariectomized mice (24). Other studies have indicated that exercise promotes osteogenic differentiation in ovariectomized rats $(25,26)$. The present study revealed that treadmill running led to an increase in the MAR and BFR/BV values in the OVX $+\mathrm{EX}$ and SHAM + EX groups compared with the OVX and SHAM groups, respectively; however, the difference between the OVX + EX and OVX groups was not significant. These findings indicated that the running exercise increased both the BMD and the bone mass in osteoporotic mice through an increase in bone formation.

IncRNAs, a recently discovered class of regulatory RNAs, have been demonstrated to have a notable role in bone metabo$\operatorname{lism}(14,16,27,28)$. The expression of $1 n c R N A s$ is responsive to different physiological and pathological stimuli (29). Previous studies have suggested that clusters of lncRNAs are aberrantly expressed during osteogenic differentiation of stem cells (30) and in ovariectomized mice (31). The pairwise comparison results [OVX vs. OVX + EX; OVX vs. SHAM; SHAM vs. $\mathrm{SHAM}+\mathrm{EX} ; \mathrm{OVX}+\mathrm{EX}$ vs. SHAM + EX] of the present study identified a total of 2,424 significantly differently expressed lncRNAs (1718 upregulated lncRNAs and 706 downregulated lncRNAs). Among them, lncRNA H19 was significantly upregulated in the OVX group following exercise training. Previous studies have indicated that lncRNA H19 is involved in tension-induced osteogenesis of stem cells (28), and that its levels are decreased in the distal femur of disuse osteoporosis rats, where it targets Dickkopf (DDK) 4 to activate the Wnt signaling pathway (32). This suggests that lncRNA H19 may also play a notable role in the functions of bone metabolism under the exercise stimulus.

IncRNAs are transcribed by RNA polymerase II and end up being localized in the nucleus, cytoplasm or in both. They have been demonstrated to regulate genome imprinting and gene expression, and also to participate in X-chromosome-silencing and other biological processes (33). Evidence suggests that lncRNAs may fulfill important regulatory roles during the development of osteoporosis $(31,34)$. LncRNA colorectal neoplasia differentially expressed is significantly upregulated in the osteoclasts of patients with postmenopausal osteoporosis (35). LncRNA maternally expressed 3 is expressed at a significantly high level in the BMSCs of postmenopausal women with osteoporosis, which leads to the upregulated expression of miR-133a-3p to inhibit osteogenic differentiation (36). Considering that estrogen deficiency increases bone turnover (37), these findings suggest that the markedly increased activity of osteoclasts observed in the present study after exercise mainly resulted from an inhibition of bone resorption in osteoporosis.

lncRNAs have a secondary structure that provides binding sites for proteins or RNAs that are involved in the regulation of transcription, translation, cell differentiation and other biological processes (38). In the present study, the enriched GO pathway terms were associated with 'cellular components' 'molecular functions' and 'biological processes'; therefore, these processes were implicated in the treatment and prevention of osteoporosis 
Table II. miRNAs targeted by the dysregulated lncRNAs.

A, Upregulated expression

\begin{tabular}{ll}
\hline IncRNA & \multicolumn{1}{c}{ miRNA target sites } \\
\hline LOC105246953 & mmu-miR-215-3p, mmu-miR-670-3p, mmu-miR-1186, mmu-miR-1186b \\
LOC102637959 & mmu-miR-15a-5p, mmu-miR-706, mmu-miR-466i-5p, mmu-miR-1187, mmu-miR-1195 \\
NONMMUT014677 & mmu-miR-669c-3p, mmu-miR-466f-3p, mmu-miR-466k, mmu-miR-574-5p, \\
& mmu-miR-466i-5p, mmu-miR-466i-3p, mmu-miR-1187, mmu-miR-466m-3p, \\
& mmu-miR-3095-3p \\
NONMMUT027251 & mmu-miR-669c-3p, mmu-miR-466f-3p, mmu-miR-574-5p, mmu-miR-466i-5p, \\
& mmu-miR-466i-3p, mmu-miR-1187, mmu-miR-466k, mmu-miR-466m-3p, \\
& mmu-miR-3095-3p \\
\hline
\end{tabular}

B, Downregulated expression

$\operatorname{lncRNA}$

rilD130079K21IPX00187K1611491

NONMMUT006626
miRNA target sites

mmu-miR-302b-3p, mmu-miR-669c-3p, mmu-miR-466i-3p, mmu-miR-669h-3p, mmu-miR-466m-3p, mmu-miR-466q

mmu-miR-328-3p, mmu-miR-3473b, mmu-miR-5128, mmu-miR-185-3p, mmu-miR-221-5p, mmu-miR-221-3p, mmu-miR-452-3p, mmu-miR-466i-5p, mmu-miR-1187, mmu-miR-3473

lncRNA, long non-coding RNA; miR/miRNA, microRNA; mmu, Mus musculus.

by exercise. IL-17A and VEGF were revealed to be enriched molecules. c-Jun N-terminal kinase is another MAPK that is involved in TNF superfamily member 11-induced osteoclast formation (39). VEGF is an angiogenesis factor expressed in osteoblast precursor cells (40), which has a key role in bone angiogenesis and osteoblastogenesis (41). The mTOR signaling pathway is one of the most important enriched pathways, and this pathway has been reported to promote osteoblast differentiation and bone mineralization in older animals (42). The NF- $\kappa \mathrm{B}$ and PI3K signaling pathways were also featured in the top 20 significantly enriched pathways. The loss or inhibition of $\mathrm{NF}-\kappa \mathrm{B}$ results in insufficient levels of osteoclastogenesis, which thereby increases bone mass in mice $(43,44)$, and PI3K is required to regulate osteoblastic differentiation of mesenchymal stem cells (45). The KEGG pathway analysis results also revealed that the expression of IncRNAs regulated by exercise were associated with the 'mTOR', 'PI3K' and 'NF- $\kappa \mathrm{B}$ ' signaling pathways, indicating that, in terms of explaining how exercise may exert its influence on osteoporosis, lncRNAs may regulate the differentiation of osteoblasts and osteoclasts mainly through these signaling pathways.

IncRNAs have been reported to act as competing endogenous RNAs for miRNAs (46), indicating that they may have an important role in miRNA-associated biological processes. The results of the present study identified that 18 lncRNAs (15 upregulated and three downregulated) were held in common between the SHAM and OVX + EX treatment groups when compared with the OVX group. Exercise might have reversed the expression of lncRNAs in the OVX group to reduce the effects of bone loss in osteoporosis. Target miRNAs were then predicted through miRNA prediction analysis to postulate the functional roles of lncRNAs in the bone. It was hypothesized that one IncRNA may interact with multiple miRNAs and, vice versa, one miRNA may connect with two or more lncRNAs. miR-15a was identified as a potential target of IncRNA LOC102637959. miR-15a is a member of the miR-15 family, which has been demonstrated to have an important role in exercise-induced bone formation by inhibiting bone morphogenetic protein (BMP) signaling in bone cells (47). This finding indicated that lncRNA LOC102637959 may be the positive regulators of osteoporosis under an exercise stimulus. In addition, a previous study revealed that silencing miR-221-3p promotes osteogenic differentiation of BMSCs through targeting insulin-like growth factor-1 to activate the ERK signaling pathway (48). Another study revealed that the knockout of miR-185-3p increases the osteogenic differentiation of osteoblasts and BMSCs, and also causes a reduction in the bone loss of osteoporotic mice by enhancing BMP signaling (49). miR-302b was also reported to activate $\mathrm{Wnt} / \beta$-catenin by degrading DKK1 to promote the differentiation of the osteoblastic cell line MC3T3-E1 (50). miR-221-3p, miR-185-3p and miR-302b were identified as the target miRNAs of downregulated lncRNAs in osteoporotic mice following exercise training, indicating that exercise may alter the expression of specific lncRNAs, thereby enhancing their inhibition of targeted miRNAs to promote bone formation. 
However, there were several limitations that must be considered both in terms of the context of the experimental design of the present study and in terms of the explanation of the results. First, only three bone samples were selected for each group for lncRNA array analysis, and the expression levels of associated lncRNAs were not verified. Therefore, the possible presence of false-negative or positive results could not be discounted. Additionally, potential roles of lncRNAs identified by microarray analysis and the association of molecules, proteins or miRNAs needs to be further confirmed. Thirdly, bone homeostasis is mediated by several types of bone cells, and therefore further studies are required to determine the function and mechanism of lncRNAs on specific cell types in the bone.

In conclusion, the present study demonstrated that the expression levels of certain lncRNAs were regulated by exercise in osteoporotic mice, and also revealed the putative functions of these lncRNAs in preventing osteoporosis. Specific lncRNAs, such as lncRNA H19 and LOC102637959, may be involved in this process. These findings have improved the current understanding of the role of exercise in regulating lncRNA expression and provided some evidence on the underlying mechanism, which should prove to be useful in terms of designing novel therapeutic interventions for the prevention and treatment of osteoporosis.

\section{Acknowledgements}

Not applicable.

\section{Funding}

The present work was supported by The National Natural Science Foundation of China (grant nos. 81572242 and 81702235), the Shanghai Key Lab of Human Sport Competence Development and Maintenance (Shanghai University of Sport; grant no. 11DZ2261100).

\section{Availability of data and materials}

The datasets generated and/or analyzed during the current study are available in the Gene Expression Omnibus repository, https://www.ncbi.nlm.nih.gov/geo/query/acc. cgi?acc $=$ GSE184226.

\section{Authors' contributions}

JG, LL, MZ and HL performed the experiments; JG and XC wrote the manuscript; YY, XT and LZ analyzed the data and prepared the figures; MW performed the target microRNA analysis and prepared the tables; $\mathrm{XC}$ and $\mathrm{JZ}$ contributed to the design of the work and supervised this entire program. JZ and $\mathrm{XC}$ confirm the authenticity of all the raw data. All authors reviewed, read and approved the final manuscript.

\section{Ethics approval and consent to participate}

The animal experiments were approved by the Ethics Committee of Shanghai University of Sport (approval no. 2015030), and all procedures were conducted in accordance with the recommendations of the ARRIVE guidelines.

\section{Patient consent for publication}

Not applicable.

\section{Competing interests}

The authors declare that they have no competing interests.

\section{References}

1. Bliuc D, Alarkawi D, Nguyen TV, Eisman JA and Center JR: Risk of subsequent fractures and mortality in elderly women and men with fragility fractures with and without osteoporotic bone density: The Dubbo Osteoporosis Epidemiology Study. J Bone Miner Res 30: 637-646, 2015.

2. Moreira LD, Oliveira ML, Lirani-Galvão AP, Marin-Mio RV, Santos RN and Lazaretti-Castro M: Physical exercise and osteoporosis: Effects of different types of exercises on bone and physical function of postmenopausal women. Arq Bras Endocrinol Metabol 58: 514-522, 2014.

3. Ma D, Wu L and He Z: Effects of walking on the preservation of bone mineral density in perimenopausal and postmenopausal women: A systematic review and meta-analysis. Menopause 20: 1216-1226, 2013

4. Hamilton CJ, Swan VJ and Jamal SA: The effects of exercise and physical activity participation on bone mass and geometry in postmenopausal women: A systematic review of pQCT studies. Osteoporos Int 21: 11-23, 2010.

5. Weaver CM, Gordon CM, Janz KF, Kalkwarf HJ, Lappe JM, Lewis R, O'Karma M, Wallace TC and Zemel BS: The National Osteoporosis Foundation's position statement on peak bone mass development and lifestyle factors: A systematic review and implementation recommendations. Osteoporos Int 27: 1281-1386, 2016.

6. Yuan Y, Chen X, Zhang L, Wu J, Guo J, Zou D, Chen B, Sun Z, Shen $C$ and Zou J: The roles of exercise in bone remodeling and in prevention and treatment of osteoporosis. Prog Biophys Mol Biol 122: 122-130, 2016

7. Chen X, Li L, Guo J, Zhang L, Yuan Y, Chen B, Sun Z, Xu J and Zou J: Treadmill running exercise prevents senile osteoporosis and upregulates the Wnt signaling pathway in SAMP6 mice. Oncotarget 7: 71072-71086, 2016.

8. Li L, Chen X, Lv S, Dong M, Zhang L, Tu J, Yang J, Zhang L, Song Y, Xu L, et al: Influence of exercise on bone remodeling-related hormones and cytokines in ovariectomized rats: A model of postmenopausal osteoporosis. PLoS One 9: e112845, 2014.

9. Brosnan CA and Voinnet O: The long and the short of noncoding RNAs. Curr Opin Cell Biol 21: 416-425, 2009.

10. Krol J, Krol I, Alvarez CP, Fiscella M, Hierlemann A, Roska B and Filipowicz W: A network comprising short and long noncoding RNAs and RNA helicase controls mouse retina architecture. Nat Commun 6: 7305, 2015.

11. Wu QY, Li X, Miao ZN, Ye JX, Wang B, Zhang F, Xu RS, Jiang DL, Zhao MD and Yuan FL: Long non-coding RNAs: A new regulatory code for osteoporosis. Front Endocrinol (Lausanne) 9: 587, 2018

12. Gong YY, Peng MY, Yin DQ and Yang YF: Long non-coding RNA H19 promotes the osteogenic differentiation of rat ectomesenchymal stem cells via Wnt/ $\beta$-catenin signaling pathway. Eur Rev Med Pharmacol Sci 22: 8805-8813, 2018.

13. Liao J, Xiao H, Dai G, He T and Huang W: Recombinant adenovirus (AdEasy system) mediated exogenous expression of long non-coding RNA H19 (lncRNA H19) biphasic regulating osteogenic differentiation of mesenchymal stem cells (MSCs). Am J Transl Res 12: 1700-1713, 2020.

14. Zhang J, Tao Z and Wang Y: Long non-coding RNA DANCR regulates the proliferation and osteogenic differentiation of human bone-derived marrow mesenchymal stem cells via the p38 MAPK pathway. Int J Mol Med 41: 213-219, 2018.

15. Jin C, Jia L, Huang Y,Zheng Y, Du N, Liu Y and Zhou Y: Inhibition of lncRNA MIR31HG Promotes Osteogenic Differentiation of Human Adipose-Derived Stem Cells. Stem Cells 34: 2707-2720, 2016. 
16. Xiaoling G, Shuaibin L and Kailu L: MicroRNA-19b-3p promotes cell proliferation and osteogenic differentiation of BMSCs by interacting with lncRNA H19. BMC Med Genet 21: 11, 2020.

17. Gao X, Ge J, Li W, Zhou W and Xu L: LncRNA KCNQ1OT1 ameliorates particle-induced osteolysis through inducing macrophage polarization by inhibiting miR-21a-5p. Biol Chem 399: 375-386, 2018.

18. Bu Y, Zheng D, Wang L and Liu J: LncRNA TSIX promotes osteoblast apoptosis in particle-induced osteolysis by down-regulating miR-30a-5p. Connect Tissue Res 59: 534-541, 2018.

19. Percie Du Sert N, Hurst V, Ahluwalia A, Alam S, Avey MT, Baker M, Browne WJ, Clark A, Cuthill IC, Dirnagl U, et al: The ARRIVE guidelines 2.0: updated guidelines for reporting animal research. BMJ Open Sci 4: e100115, 2020.

20. Inada M, Matsumoto C and Miyaura C: Animal models for bone and joint disease. Ovariectomized and orchidectomized animals. Clin Calcium 21: 164-170, 2011 (In Japanese).

21. Høydal MA, Wisløff U, Kemi OJ and Ellingsen O: Running speed and maximal oxygen uptake in rats and mice: Practical implications for exercise training. Eur J Cardiovasc Prev Rehabil 14: 753-760, 2007.

22. Deckard C, Walker A and Hill BJF: Using three-point bending to evaluate tibia bone strength in ovariectomized young mice. J Biol Phys 43: 139-148, 2017.

23. Zhang L, Chen X, Wu J, Yuan Y, Guo J, Biswas S, Li B and Zou J: The effects of different intensities of exercise and active vitamin $\mathrm{D}$ on mouse bone mass and bone strength. J Bone Miner Metab 35: 265-277, 2017

24. Zhang M, Ishikawa S, Inagawa T, Ikemoto H, Guo S, Sunagawa M and Hisamitsu T: Influence of mechanical force on bone matrix proteins in ovariectomised mice and osteoblast-like MC3T3-E1 Cells. In Vivo 31: 87-95, 2017.

25. Bu S, Chen Y, Wang S, Zhang F and Ji G: Treadmill training regulates $\beta$-catenin signaling through phosphorylation of GSK-3 $\beta$ in lumbar vertebrae of ovariectomized rats. Eur J Appl Physiol 112: 3295-3304, 2012.

26. Liu M, Zhong C, He RX and Chen LF: Icariin associated with exercise therapy is an effective treatment for postmenopausal osteoporosis. Chin Med J (Engl) 125: 1784-1789, 2012.

27. Shang G, Wang Y, Xu Y,Zhang S, Sun X, Guan H,Zhao X, Wang Y, Li Y and Zhao G: Long non-coding RNA TCONS_00041960 enhances osteogenesis and inhibits adipogenesis of rat bone marrow mesenchymal stem cell by targeting miR-204-5p and miR-125a-3p. J Cell Physiol 233: 6041-6051, 2018.

28. Wu J, Zhao J, Sun L, Pan Y, Wang H and Zhang WB: Long non-coding RNA H19 mediates mechanical tension-induced osteogenesis of bone marrow mesenchymal stem cells via FAK by sponging miR-138. Bone 108: 62-70, 2018

29. Yang Y, Yujiao W, Fang W, Linhui Y, Ziqi G, Zhichen W, Zirui W and Shengwang W: The roles of miRNA, lncRNA and circRNA in the development of osteoporosis. Biol Res 53: 40, 2020.

30. Huang G, Kang Y, Huang Z, Zhang Z, Meng F, Chen W, Fu M, Liao $\mathrm{W}$ and Zhang Z: Identification and characterization of long non-coding RNAs in osteogenic differentiation of human adipose-derived stem cells. Cell Physiol Biochem 42: 1037-1050, 2017.

31. Hao L, Fu J, Tian Y and Wu J: Systematic analysis of lncRNAs, miRNAs and mRNAs for the identification of biomarkers for osteoporosis in the mandible of ovariectomized mice. Int J Mol Med 40: 689-702, 2017.

32. Li B, Liu J, Zhao J, Ma JX, Jia HB, Zhang Y, Xing GS and Ma XL: LncRNA-H19 modulates Wnt/ $\beta$-catenin signaling by targeting Dkk4 in hindlimb unloaded rat. Orthop Surg 9: 319-327, 2017.

33. Wei CW, Luo T, Zou SS and Wu AS: The role of long noncoding RNAs in central nervous system and neurodegenerative diseases. Front Behav Neurosci 12: 175, 2018.

34. Zhao N, Zeng L, Liu Y, Han D, Liu H, Xu J, Jiang Y, Li C, Cai T, Feng $\mathrm{H}$, et al: DLX3 promotes bone marrow mesenchymal stem cell proliferation through H19/miR-675 axis. Clin Sci (Lond) 131: 2721-2735, 2017.
35. Li W, Zhu HM, Xu HD, Zhang B and Huang SM: CRNDE impacts the proliferation of osteoclast by estrogen deficiency in postmenopausal osteoporosis. Eur Rev Med Pharmacol Sci 22: 5815-5821, 2018

36. Wang Q, Li Y, Zhang Y, Ma L, Lin L, Meng J, Jiang L, Wang L, Zhou P and Zhang Y: LncRNA MEG3 inhibited osteogenic differentiation of bone marrow mesenchymal stem cells from postmenopausal osteoporosis by targeting miR-133a-3p. Biomed Pharmacother 89: 1178-1186, 2017.

37. Cauley JA: Estrogen and bone health in men and women. Steroids 99: 11-15, 2015.

38. Wapinski $\mathrm{O}$ and Chang HY: Long noncoding RNAs and human disease. Trends Cell Biol 21: 354-361, 2011.

39. Linder M, Hecking M, Glitzner E, Zwerina K, Holcmann M, Bakiri L, Ruocco MG, Tuckermann J, Schett G, Wagner EF, et al: EGFR controls bone development by negatively regulating mTOR-signaling during osteoblast differentiation. Cell Death Differ 25: 1094-1106, 2018.

40. Ruocco MG, Maeda S, Park JM, Lawrence T, Hsu LC, Cao Y, Schett G, Wagner EF and Karin M: I\{kappa\}B kinase (IKK) \{beta\}, but not IKK\{alpha\}, is a critical mediator of osteoclast survival and is required for inflammation-induced bone loss. J Exp Med 201: $1677-1687,2005$.

41. Franzoso G, Carlson L, Xing L, Poljak L, Shores EW, Brown KD, Leonardi A, Tran T, Boyce BF and Siebenlist U: Requirement for NF-kappaB in osteoclast and B-cell development. Genes Dev 11: 3482-3496, 1997.

42. Youssef A and Han VKM: Regulation of osteogenic differentiation of placental-derived mesenchymal stem cells by insulin-like growth factors and low oxygen tension. Stem Cells Int 2017: 4576327, 2017.

43. Kang H, Yan Y, Jia P, Yang K, Guo C, Chen H, Qi J, Qian N, Xu X, Wang F, et al: Desferrioxamine reduces ultrahigh-molecular-weight polyethylene-induced osteolysis by restraining inflammatory osteoclastogenesis via heme oxygenase-1. Cell Death Dis 7: e2435, 2016.

44. Mayr-Wohlfart U, Waltenberger J, Hausser H, Kessler S, Günther KP, Dehio C, Puhl W and Brenner RE: Vascular endothelial growth factor stimulates chemotactic migration of primary human osteoblasts. Bone 30: 472-477, 2002.

45. HaDuong JH, Blavier L, Baniwal SK, Frenkel B, Malvar J, Punj V, Sposto R and DeClerck YA: Interaction between bone marrow stromal cells and neuroblastoma cells leads to a VEGFA-mediated osteoblastogenesis. Int J Cancer 137: 797-809, 2015.

46. Xia T, Liao Q, Jiang X, Shao Y, Xiao B, Xi Y and Guo J: Long noncoding RNA associated-competing endogenous RNAs in gastric cancer. Sci Rep 4: 6088, 2014.

47. Grünhagen J, Bhushan R, Degenkolbe E, Jäger M, Knaus P, Mundlos S, Robinson PN and Ott CE: MiR-497 195 cluster microRNAs regulate osteoblast differentiation by targeting BMP signaling. J Bone Miner Res 30: 796-808, 2015.

48. Gan K, Dong GH, Wang N and Zhu JF: miR-221-3p and miR-222-3p downregulation promoted osteogenic differentiation of bone marrow mesenchyme stem cells through IGF-1/ERK pathway under high glucose condition. Diabetes Res Clin Pract 167: $108121,2020$.

49. Cui Q, Xing J, Yu M, Wang Y, Xu J, Gu Y, Nan X, Ma W, Liu H and Zhao H: Mmu-miR-185 depletion promotes osteogenic differentiation and suppresses bone loss in osteoporosis through the Bgn-mediated BMP/Smad pathway. Cell Death Dis 10: 172, 2019.

50. Wu Z, Zhang Y, Yang Z, Zhu Y, Xie Y, Zhou F and Cai L: Elevation of miR-302b prevents multiple myeloma cell growth and bone destruction by blocking DKK1 secretion. Cancer Cell Int 21: 187, 2021

This work is licensed under a Creative Commons Attribution-NonCommercial-NoDerivatives 4.0 International (CC BY-NC-ND 4.0) License. 